Eastern-European Journal of Medical Humanities and Bioethics ISSN: 2559-7604

Covered in: CrossRef; RePEC; CEEOL; KVK; WorldCat; Google Scholar

2018, Volume 2, Issue 1, pages: 101-103 | doi: https://doi.org/10.18662/eejmhb/10

\title{
Interferences between Clinical Practice and Public Health
}

\section{Letter to the Editor on a} Scientific Event

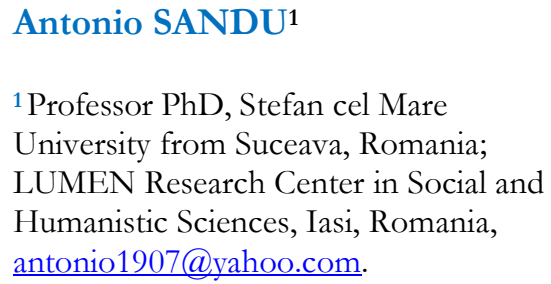

How to cite: Sandu, A. (2018). Interferences between Clinical Practice and Public Health. Eastern-European Journal of Medical Humanities and Bioetbics, 2(1), 101-103. https://doi.org/10.18662/eejmhb/10 
This article consists of reflections on the habilitation thesis of Mr. Liviu Oprea, MD, MA, PhD Associate Professor, entitled "Research on ethical issues at the interface between clinical practice and public health", defended at the "Gr. T. Popa" University of Medicine and Pharmacy of Iasi April 20, 2018.

We are facing a transdisciplinary habilitation thesis, which proves a thorough knowledge of the ethical issues both in the field of clinical practice and in the field of public health.

\section{Strong points:}

- professional experience of the candidate:

- Master of Arts in Bioethics program at Case Western Reserve University in Cleveland, Ohio, United States, (top 150)

- PhD degree in Medicine from Adelaide University, Adelaide, Australia (top 150).

- transdisciplinary approach: bioethics, public health, social sciences and behavioural sciences, which allows him to conduct a thorough research on the issue of the doctor-patient relationship, both from a clinical perspective, and from that of public health.

- I can't fail to emphasize the fact that Liviu Oprea contributed to the development of numerous researchers, among which a few very valuable ones nationally and internationally, in the field of bioethics and public health in Romania, within the Center for Ethics and Health-related Policies (Postdoctoral studies in Health Policy (POSDRU/89/1.5/S/61879) he had coordinated at Gr. T. Popa University of Medicine and Pharmacy, and in which I had the honor to be a postdoctoral researcher. Other two projects, Program of Excellence in Doctoral and Postdoctoral Research in Chronic Diseases (POSDRU/159/1.5/S/133377), The East European Network of Excellence for Research in the Field of Bioethics and Public Health.

- experience in research

- use of methods of social research, both quatitative and qualitative in the field of ethics, bringing ethics, from an epistemological point of view, in the area of social sciences associated with medical practices.

- contributions to the field of studying the doctor-patient relationship in the context of care of chronic diseases: study on the issue of mutual trust between the doctor and the patient, key element of this relationship and the communicational environment in which the evidence-based medical practice can be implemented. As far as the transfer (construction) of responsibility for self-care by the patient is concerned, we consider the issue of the doctor's trust in the patient, which co-exists with the - more widely researched - of the patient in the doctor. The contributions of the candidate aim to cover a part of the gaps noticed by the author in the literature, namely on the mechanisms of constructing and maintaining this relationship of trust between the two actors 
of the therapeutic relationship, and how does the trust from one's part influences the trust of the other part. Synthesizing the mechanisms identified in the literature, the author emphasizes loyality, the relationship of trust built in time, the communication focused on patient, the patient's honesty and trust in the seriousness of the medical act. These mechanisms are investigated by the candidate in the particular context given by two chronic diseases: the irritable bowel syndrome, and the diabetes. As a mechanism of creating and maintaining mutual trust, it is identified the continuous therapeutic success, which proves the patient the efficiency of care and the competence of the doctor, generating the patient's loyalty towards his doctor. Another mechanism identified is the richness of the dialogue between the two parts, the patient interpreting an increased volume of questions from the doctor as being a sign of real interest for the diagnosis and for a correct treatment, and the doctor interpreting the same high volume of questions from the patient as being interest for the process of self-care. The patterns of communication present in the dialogue between the two parts are also significant in the construction of mutual trust in the case of anxious patients. The medical investigations the patients are subject to also represents a source of trust, precisely due to the value of the results (in the sense of evidence-based medical practice).

- contributions to the ethical analysis of the informal payments in the health-care system: the issue of informal payments in the medical system affects the quality of the act of care, being considered as affecting the medical systems in countries under transition. The candidate clearly highlights a series of characteristics of informal payments based on the people involved (patients and caregivers, and doctors), the moment of payment - before, during or after the medical act, the form of payment (cash, goods or services). The author analyses the informal payments in the medical system from an ethical perspective, thus filling an existing lack in the romanian (bio)ethical scientific literature. The informal payments are usually approached in a journalistic manner, seldom emphasizing on the illegitimate and unethical nature. In order to delineate from the journalistic practice of analysing the informal payments, the author develops an evaluation grid of their impact, having a series of components: defining informal payments, the ways of achieving it, the payment time, reasons and consequences of informal payments to the patient-doctor relationship.

\section{References}

Oprea, L. (2018). Research on ethical issues at the interface between clinical practice and public health, habilitation thesis, Universitatea de Medicină şi Farmacie Gr. T Popa din Iaşi, 20.04.2018. Available at http://www.umfiasi.ro/ScoalaDoctorala/ Conf $\% 20$ univ $\% 20 \mathrm{dr} \% 200$ prea $\% 20$ Liviu/02_Teza abilitare.pdf 